\title{
Comparison Between Nonlinear and Linear Spectrographic Techniques for the Complete Characterization of High Bit-Rate Pulses Used in Optical Communications
}

\author{
Benn C. Thomsen, Michaël A. F. Roelens, Regan T. Watts, and David J. Richardson
}

\begin{abstract}
Two spectrographic techniques for the complete characterization (amplitude and phase) of optical pulses with durations commensurate with high bit-rate communications systems (2-30 ps) are experimentally compared. We show that a highly sensitive linear sampling technique utilizing an electroabsorption modulator gives accurate results when compared to a nonlinear sampling technique based on second-harmonic generation over a range of pulse durations from 2-33 ps.
\end{abstract}

Index Terms-High-speed transmission, optical communications, optical pulse characterization.

\section{INTRODUCTION}

$\mathbf{T}$ HE characterization of the amplitude and phase of short ( 1-30 ps) low-power optical pulses such as those envisioned for use in high bit-rate communication systems is challenging. Direct electronic measurements are limited by the achievable sampling bandwidth, while the much higher bandwidth nonlinear optical techniques suffer from a lack of sensitivity. Such measurements are increasingly important for the design and monitoring of optical systems that operate at $40 \mathrm{~Gb} / \mathrm{s}$ and beyond where the performance is very sensitive to the pulse amplitude and phase and the detailed characteristics of the transmission system itself. In order to overcome the electronic bandwidth limitation, a number of optical techniques to completely characterize such pulses have been developed including spectrographic nonlinear techniques [1], and nonlinear and linear interferometric techniques [2], [3].

In this letter, we compare two experimental implementations of the spectrographic techniques that are most suited to the measurement of optical pulses that are used in high bit-rate $1.5-\mu \mathrm{m}$ optical communication systems. The experimental implementations considered are second-harmonic-generation-based frequency resolved optical gating (SHG-FROG) developed by Trebino and Delong [1], and the recently reported linear-spectrographic technique developed by Dorrer and Kang [4].

Manuscript received on March 9, 2005. Revised on May 24, 2005.

B. C. Thomsen is with the Optical Networks Group, Department of Electronic and Electrical Engineering, University College London, London WC1E 7JE, U.K. (e-mail: bthomsen@ee.ucl.ac.uk).

M. A. F. Roelens and D. J. Richardson are with the Optoelectronics Research Centre, University of Southampton, Southampton SO17 1BJ, U.K. (e-mail: mafr@orc.soton.ac.uk; djr@orc.soton.ac.uk).

R. T. Watts is with the Department of Physics, The University of Auckland, Auckland, New Zealand (e-mail: r.watts@ southernphotonics.com).

Digital Object Identifier 10.1109/LPT.2005.853261

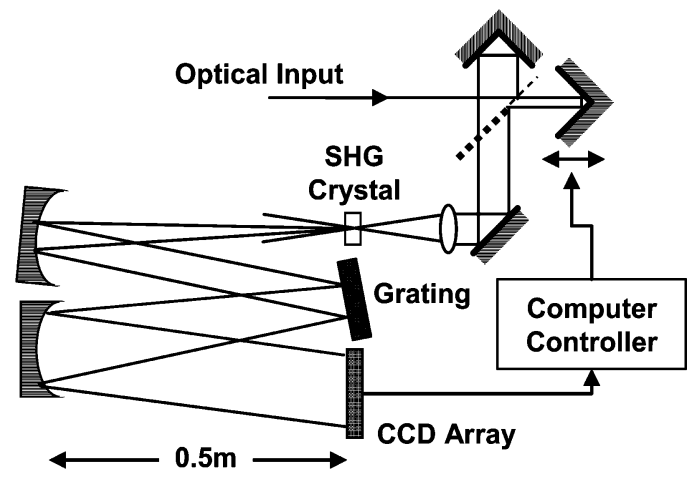

Fig. 1. Experimental setup for SHG-FROG measurement.

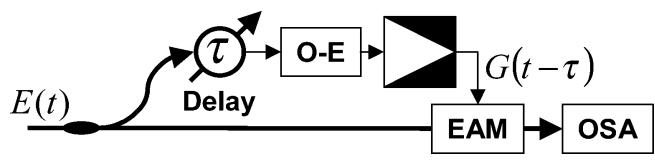

Fig. 2. Linear-spectrogram setup.

The FROG technique has been used for ultrashort pulse characterization in a range of applications [5], [6]. Its versatility lies in the fact that the pulse itself acts as its own gate, thus, the gate duration is optimally tailored to the pulse duration, with a measurement bandwidth only limited by the phase matching bandwidth of the nonlinear process. However, the use of a nonlinear optical process results in low sensitivity and high polarization sensitivity. Dorrer and Kang recently demonstrated a variation on this pulse characterization technique that employs linear optical gating using an electroabsorption modulator (EAM) [4]. This linear-spectrographic technique has much greater sensitivity, is much less polarization sensitive, and it does not suffer from the temporal ambiguity in the measured fields that is associated with SHG-FROG. Recently, Dorrer demonstrated the characterization of pulses as short as $900 \mathrm{fs}$ using gate of $30 \mathrm{ps}$ in duration [7].

Here we present the results from an experimental comparison between the well-established SHG-FROG technique and the linear-spectrographic technique for pulse durations ranging from 33 to 2 ps in order to verify the accuracy of this technique. 
(a)

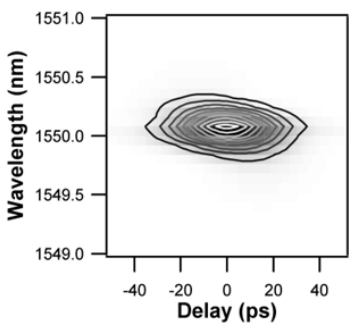

(b)



(c)

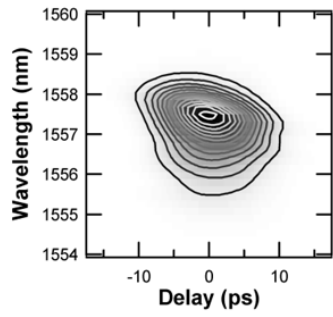

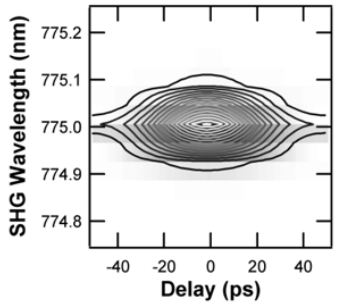
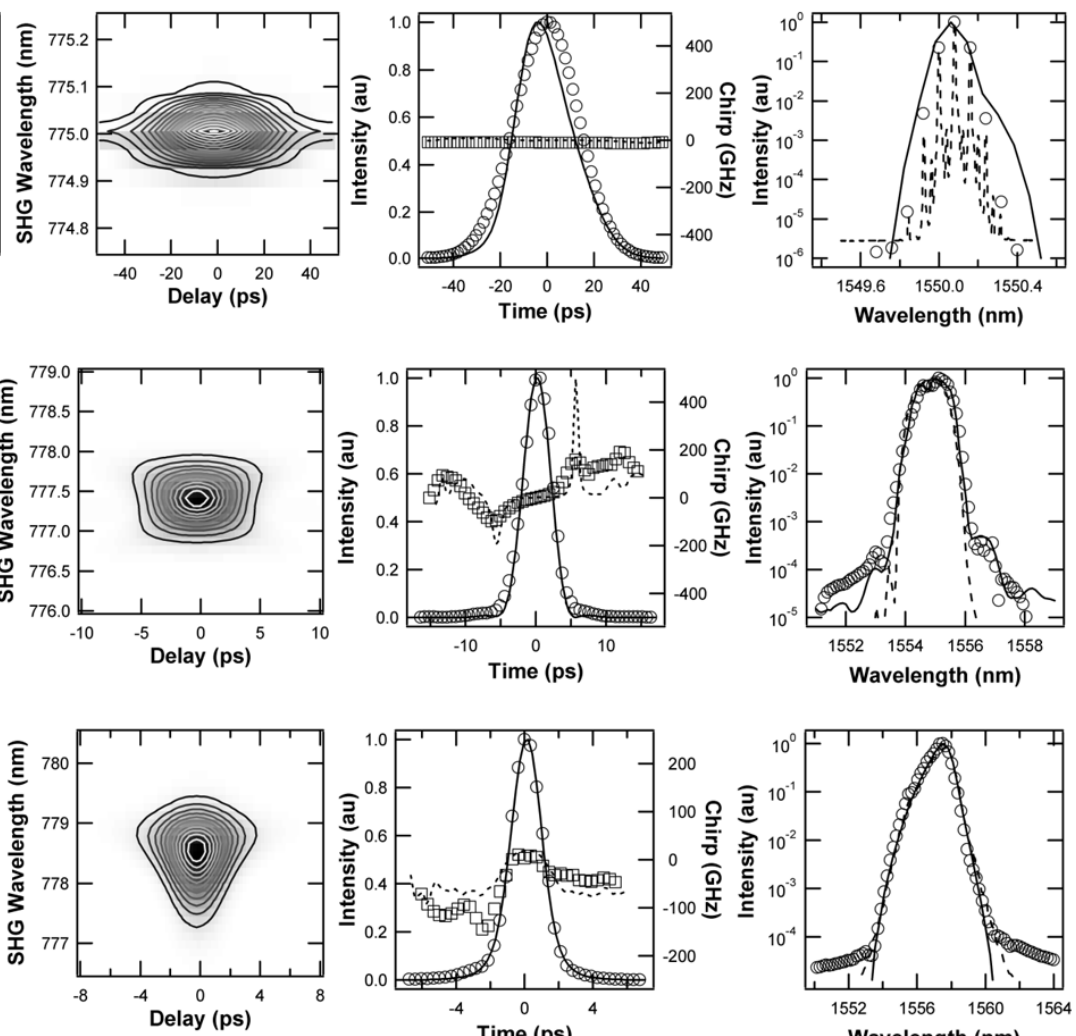



linear and SHG-FROG spectrograms, the retrieved temporal intensity and chirp (Linear: circles and squares; SHG-FROG: solid and dashed lines) and the retrieved spectral intensity chirp (Linear: circles; SHG-FROG: solid line) compared to the independently measured spectrum (dotted line).

\section{EXPERIMENTAL SETUP}

The experimental setup for obtaining SHG-FROG measurements is shown in Fig. 1. The pulse and its time delayed replica are recombined in a nonlinear crystal whose SHG output is proportional to the product of the two fields. This signal is then spectrally resolved as a function of the temporal delay. The SHG-FROG used for these measurements employs a Czerny-Turner type spectrometer that provides a bandpass resolution of $20.35 \mathrm{GHz}$ or $0.04 \mathrm{~nm}$ at the SHG wavelength of around $775 \mathrm{~nm}$ for pulses in the 1550-nm region. This FROG apparatus has a previously reported sensitivity of $500 \mathrm{~mW}^{2}$ [8]. The temporal intensity and phase is then retrieved from the experimentally obtained spectrogram using an iterative numerical algorithm [9].

Fig. 2 shows the experimental setup for measuring linear spectrograms. In this implementation, an EAM (Corning) is used to temporally gate the optical pulses. This setup utilized a self-referencing scheme that also minimizes the gating window duration. Part of the optical pulse stream is electronically detected and amplified to $4 \mathrm{Vp}$-p. This produces a short electrical pulse of around $30 \mathrm{ps}$ in duration (limited by the $20-\mathrm{GHz}$ bandwidth of the electronics) that is used to open a gating window in the EAM. Gating window durations from 10 to $16 \mathrm{ps}$ are obtained by adjusting the bias voltage of the EAM from -6.5 to $-4 \mathrm{~V}$. In the results presented here, the bias was adjusted to ensure that the gate duration was different to that of the pulses under test to ensure any potential ambiguities in the retrieval process were avoided. The remaining optical signal is gated by the EAM and the output is spectrally resolved on an optical spectrum analyzer (OSA) as a function of the relative delay introduced between the optical signal and the gate using a computer controlled optical delay line. The OSA has a spectral resolution of $1.25 \mathrm{GHz}$ or $0.01 \mathrm{~nm}$ at $1550 \mathrm{~nm}$. The pulse electric field and the gate are numerically retrieved using the same two-dimensional blind deconvolution algorithm as for the SHG-FROG.

Dorrer et al. have demonstrated this technique for pulse energies as low as $10 \mathrm{fJ}$ at $10 \mathrm{~Gb} / \mathrm{s}$ [4]. For a sensitivity comparison, the SHG-FROG apparatus used here would require a pulse energy of $707 \mathrm{fJ}$ at $10 \mathrm{~Gb} / \mathrm{s}$ assuming a pulsewidth of 10 ps. This considerably improved sensitivity over SHGFROG arises because it does not require the use of an optical nonlinearity. The sensitivity of the SHG-FROG scales with the peak power average power product whereas the sensitivity of the linear-spectrogram technique scales linearly with average power. To accurately characterize the $10-\mathrm{GHz}$ repetition rate pulses measured in this letter, it is necessary to have a spectral resolution sufficient to sample at the Nyquist frequency of 10 GHz. Thus, the spectrograms obtained with the SHG-FROG apparatus (spectral resolution $20.35 \mathrm{GHz}$ ) are all under sampled while the resolution of the OSA $(1.25 \mathrm{GHz})$ in the linear apparatus allows for sampling at the Nyquist frequency. The under sampling will result in increased measurement errors particularly for pulses with low spectral bandwidths. This makes the linear-spectrogram technique ideally suited to the 
measurement of low peak power and low spectral bandwidth pulses. The linear-spectrogram is also much simpler to set up and more compact and robust than the SHG-FROG since all of the parts are fiber coupled and can simply be spliced together. However, the all-fiber construction of the EAM-FROG makes it unsuitable for subpicosecond pulses as the dispersive and nonlinear effects of the fiber will distort the results.

\section{RESULTS AND DISCUSSION}

To determine the experimental range of applicability for the two techniques, a series of measurements were taken on a set of pulses with durations ranging from 2 to $32 \mathrm{ps}$. The results of these measurements are presented in Fig. 3, where the columns (from left to right) are the measured EAM-FROG and SHG-FROG spectrograms, and retrieved temporal and spectral fields. Fig. 3(a) shows the results for a 10-GHz 32-ps pulse obtained by pulse carving with an overdriven lithium niobate Mach-Zehnder modulator [10]. Both retrievals were carried out on a $64 \times 64$ grid. The retrieval for the linear-spectrogram has a root mean square (rms) error of $<0.005$ and shows excellent agreement with the independently measured spectrum indicating a good retrieval. However, the SHG-FROG retrieval has a larger rms error of 0.05 and the spectrum retrieved from this measurement shows poor agreement with the measured spectrum resulting in a retrieved temporal pulse that is shorter in duration than it should be. This mismatch arises from the under sampling of the spectrogram. To quantify the effect of this spectral mismatch on the retrieved temporal intensity the retrieved spectral intensity is replaced with the measured spectral intensity and the resulting complex field is numerically Fourier transformed to calculate the complex temporal field. The resulting mismatch in the time domain is then quantified by calculating the rms error between the calculated pulse intensity and the original retrieved pulse intensity. The SHG-FROG measurement gives an rms error of 0.1 compared to an error of 0.033 for the linear spectrogram. The SHG-FROG is not able to correctly measure these pulses because the spectral resolution (20.35 GHz) of the 0.5-m spectrometer employed in this setup is insufficient to completely resolve the SHG signal. While this is not an intrinsic limitation of the SHG-FROG technique as it can be resolved by increasing the resolution of the spectrometer, the increase in required real estate and complexity make this unattractive.

Fig. 3(b) and (c) shows the results, both taken on a $128 \times 128$ grid, from a 10-GHz 5-ps pulse generated from a linearly compensated gain-switched distributed feedback laser diode [11] and 10-GHz 2-ps pulses from a harmonically mode-locked fiber laser. Here, both the EAM- and SHG-FROG techniques are able to accurately characterize the pulses as evidenced by, the rms retrieval error of $<0.005$ for both measurements, the agreement between the retrieved temporal intensity and chirp and the agreement between the retrieved spectra and the independently measured spectra for both techniques. Here, the rms error in the temporal intensity arising from the mismatch between the retrieved spectrum and the measured spectrum for the 5-ps gain-switched laser pulse is 0.015 and 0.004 and for the 2-ps mode-locked fiber laser 0.008 and 0.005 for the SHG-FROG and the linear spectrogram, respectively. While the SHG-FROG spectrogram is still under sampled as these results show, the broader spectral bandwidth of these shorter pulses reduces the effects of the under sampling.

\section{CONCLUSION}

We have shown that the resolution and sensitivity provided by an EAM-based linear-spectrographic technique utilizing a standard communication grade OSA, allows for the accurate characterization of low power pulses with durations ranging from 2 to 32 ps. In comparison, the SHG-FROG apparatus provided accurate characterization for the 2 and 5 ps pulses but produced significant measurement errors for the 32-ps pulse as a result of the under sampling due to insufficient spectrometer resolution.

\section{REFERENCES}

[1] R. Trebino, K. W. DeLong, D. N. Fittinghoff, M. A. Krumbügel, B. A. Richman, and D. J. Kane, "Measuring ultrashort laser pulses in the time frequency domain using frequency-resolved optical gating," Amer. Inst. Phys., Rev. Sci. Instrum., vol. 68, pp. 3277-3295, 1997.

[2] C. Iaconis and I. A. Walmsley, "Self-referencing spectral interferometry for measuring ultrashort optical pulses," IEEE J. Quantum Electron., vol. 35, no. 4, pp. 501-509, Apr. 1999.

[3] P. Kockaert, M. Haeltermannm, P. Emplit, and C. Froehly, "Complete characterization of (ultra)short optical pulses using fast linear detectors," IEEE J. Sel. Topics Quantum Electron., vol. 10, no. 1, pp. 206-212, Jan./Feb. 2004.

[4] C. Dorrer and I. Kang, "Simultaneous temporal characterization of telecommunication optical pulses and modulators by use of spectrograms," Opt. Lett., vol. 27, pp. 1315-1317, 2002.

[5] R. Trebino, Frequency-Resolved Optical Gating: The Measurement of Ultrashort Laser Pulses. Boston, MA: Kluwer, 2002.

[6] J. M. Dudley, L. P. Barry, J. D. Harvey, M. D. Thomson, B. C. Thomsen, P. G. Bollond, and R. Leonhardt, "Complete characterization of ultrashort pulse sources at $1550 \mathrm{~nm}$," IEEE J. Quantum Electron., vol. 35, no. 4, pp. 441-450, Apr. 1999.

[7] C. Dorrer, "Investigation of the spectrogram technique for the characterization of picosecond optical pulses," in Optical Fiber Communication Conf. on CD-ROM, Washington, DC, 2005, Paper OTuB3.

[8] B. C. Thomsen, D. A. Reid, R. T. Watts, L. P. Barry, and J. D. Harvey, "Characterization of 40-Gbit/s pulses generated using a lithium niobate modulator at $1550 \mathrm{~nm}$ using frequency resolved optical gating," IEEE Trans. Instrum. Meas., vol. 53, no. 1, pp. 186-191, Feb. 2004.

[9] D. J. Kane, "Recent progress toward real-time measurement of ultrashort laser pulses," IEEE J. Quantum Electron., vol. 35, no. 4, pp. 421-431, Apr. 1999

[10] A. Sahara, T. Komukai, E. Yamada, N. Nakazawa, and 2001, "40 Gbit/s return-to-zero transmission over $500 \mathrm{~km}$ of standard fiber using chirped fiber Bragg gratings with small group delay ripples," in Tech. Dig. Optical Fiber Communication Conf., Anaheim, CA, 2001, pp. ThF5-1-ThF5-3.

[11] L. Chusseau and C. Kazmierski, "Optimum linear pulse compression of a gain switched $1.5 \mu \mathrm{m}$ DFB laser," IEEE Photon. Technol. Lett., vol. 6 , no. 1, pp. 24-26, Jan. 1994. 